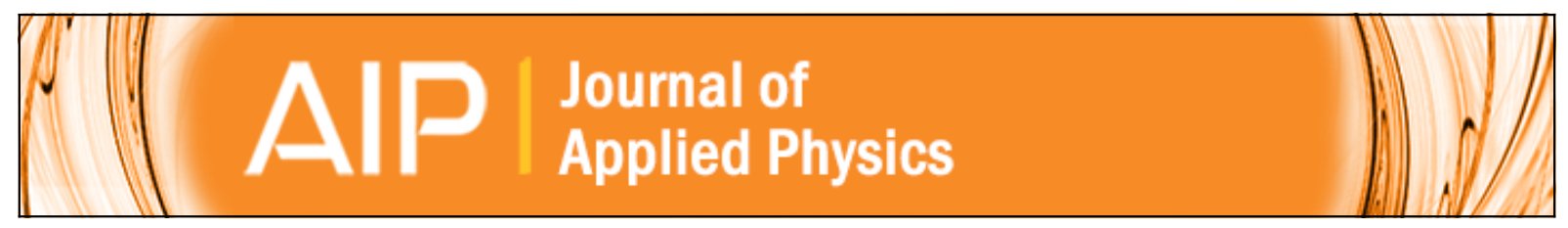

\title{
Disorder effects in the one-dimensional Anderson lattice model
}

Feng Chen and Nicholas Kioussis

Citation: Journal of Applied Physics 85, 5330 (1999); doi: 10.1063/1.370242

View online: http://dx.doi.org/10.1063/1.370242

View Table of Contents: http://scitation.aip.org/content/aip/journal/jap/85/8?ver=pdfcov

Published by the AIP Publishing

\section{Articles you may be interested in}

Effect of f-band dispersion on the magnetic properties of periodic Anderson lattice model

J. Appl. Phys. 95, 7195 (2004); 10.1063/1.1676024

A simple approach to Anderson localization in one-dimensional disordered lattices

Am. J. Phys. 72, 226 (2004); 10.1119/1.1593660

Phase diagram of the one-dimensional periodic Anderson model

J. Appl. Phys. 89, 7180 (2001); 10.1063/1.1357845

One-dimensional heavy fermion lattice model

J. Appl. Phys. 85, 5335 (1999); 10.1063/1.370244

Size and dimensionality effect in single-impurity Anderson model

J. Appl. Phys. 83, 6429 (1998); 10.1063/1.367667

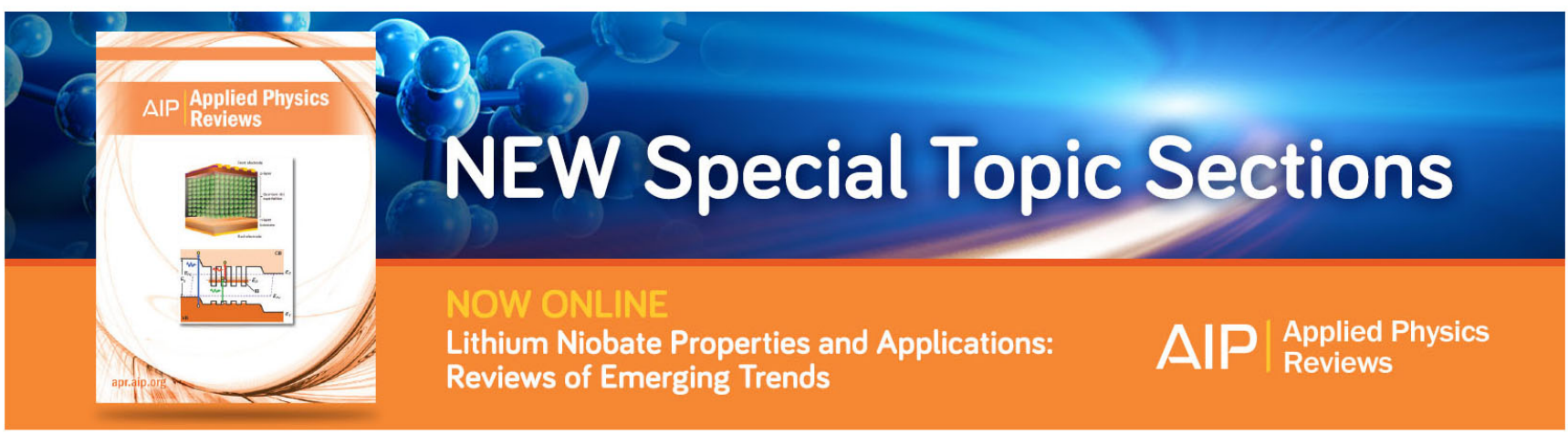




\title{
Disorder effects in the one-dimensional Anderson lattice model
}

\author{
Feng Chen and Nicholas Kioussis \\ Department of Physics and Astronomy, California State University, Northridge, California 91324-8268
}

We have studied the one-dimensional periodic, symmetric Anderson model at half filling in the presence of disorder using finite-temperature quantum Monte Carlo techniques. We have considered the disorder of both the hybridization between the local $f$-orbitals and the conduction electrons and the local $f$-site energy, using a uniform distribution of width $\Delta$. The $f$-orbital local magnetic moment, the static magnetic susceptibility, the charge susceptibility, and the nearest-neighber magnetic correlation function have been calculated as a function of the disorder distribution width $\Delta$. We find that the hybridization disorder has a dramatical effect on the low-temperature magnetic properties giving rise to a non-Fermi liquid behavior, and that the magnetic susceptibility can be scaled by a power law with an exponent which is in excellent agreement with experiment. On the other hand, disorder of the local $f$-orbital energy level does not show a non-Fermi liquid behavior. (c) 1999 American Institute of Physics. [S0021-8979(99)18008-3]

The periodic Anderson model is thought to describe the heavy-fermion systems. In the last several years a new class of heavy-fermion materials has been found. These materials exhibit non-Fermi-liquid (NFL) behavior in their physical properties at low temperatures. In several cases of heavyfermion compounds, such as $\mathrm{Y}_{1-x} \mathrm{U}_{x} \mathrm{Pd}_{3},{ }^{1}$ and $\mathrm{Ce}_{1-x} \mathrm{Th}_{x} \mathrm{RhSb}^{2}$ NFL behavior occurs only when the $f$-electron materials, consisting primarily of $\mathrm{Ce}$ or $\mathrm{U}$ intermetallics, have been alloyed with a nonmagnetic element. One of the possible theoretical explanations is the presence of the disorder in the impurity lattice systems, which presents an additional challenge that has only recently started to be addressed. ${ }^{3-5}$

We present here the first investigation of the effects of disorder on the magnetic properties of the one-dimensional periodic Anderson model (PAM) at half filling using quantum Monte Carlo techniques. ${ }^{6}$ The quantum Monte Carlo calculations treat both the Ruderman-Kittel-KasuyaYosida (RKKY) and the Kondo interactions on an equal footing, and allow one to treat both the weak and strong disorder regime.

The Hamiltonian for the one-dimensional PAM with disorder is

$$
\begin{aligned}
H= & -t \sum_{i, \sigma}\left(c_{i, \sigma}^{+} c_{i+1, \sigma}+H . c .\right)+E^{c} \sum_{i, \sigma} n_{i \sigma}^{c} \\
& +\sum_{i, \sigma} V_{i}\left(f_{i, \sigma}^{+} c_{i, \sigma}+H . c .\right) \\
& +U_{f} \sum_{i}\left(n_{i \uparrow}^{f}-\frac{1}{2}\right)\left(n_{i \downarrow}^{f}-\frac{1}{2}\right)+\sum_{i, \sigma} E_{i}^{f} n_{i \sigma}^{f} .
\end{aligned}
$$

The important thing to notice in Eq. (1) is that, unlike the usual PAM, the local $f$-site parameters $V_{i}$ or $E_{i}^{f}$ are taken here to be random numbers distributed according to uniform distributions of width $2 \Delta$ centered at $V_{0}$ or $E_{0}^{f}$, respectively. In the following, we will set $t=1$ and consider the half filling $\left(E^{c}=0\right)$ and symmetric $\left(E_{0}^{f}=0\right)$ case.
We have employed a finite-temperature quantum Monte Carlo technique with an exact updating procedure. The calculated physical observables are averaged over a sufficient number of disorder realizations. We first study the case of disorder in hybridization. In Fig. 1 we plot the impurity magnetic moment $\sigma$, the susceptibility $T \chi$, the charge compressibility $T \kappa$, and the nearest-neighbor spin correlation function $C(i, i+1)$ as a function of the disorder width $\Delta$ at low temperatures, $\beta=8$. We find that $\sigma$ increases as $\Delta$ increases. This result shows that the number of the unquenched local spins at low temperatures increases with disorder. Similarly, $T \chi$, which measures the "effective" magnetic moment, increases with increasing disorder width. The low value of the effective moment for $\Delta=0$ at low temperatures indicates that the magnetic correlations act to screen the $f$-site moment

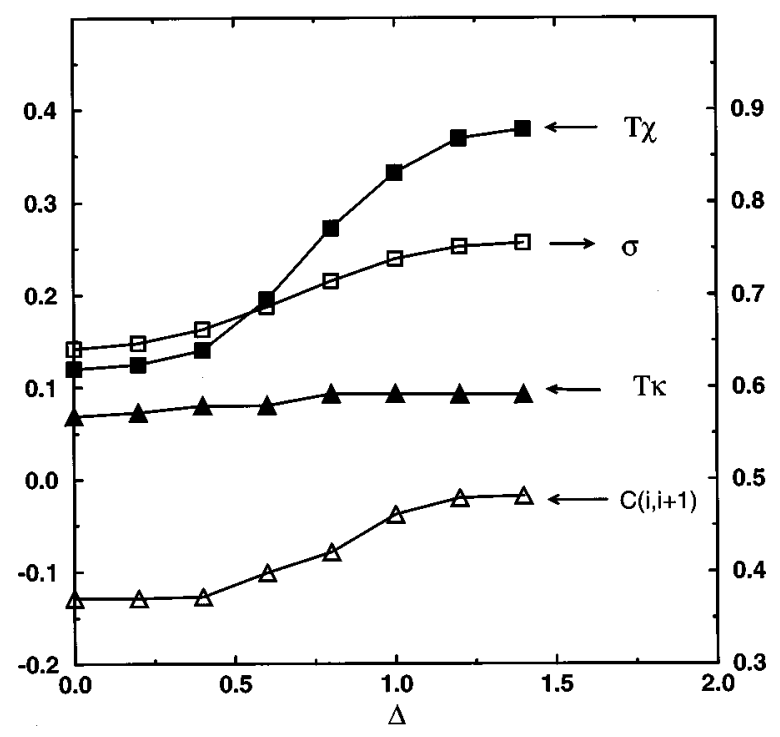

FIG. 1. Square of the $f$-orbital local moment $\sigma$ (open squares), the $f$ magnetic susceptibility $T \chi$ (solid squares), the charge susceptibility $T \kappa$ (solid triangles), and the nearest-neighbor $f-f$ spin correlation function $C(i, i$ +1 ) (open triangles) as a function of the hybridization-disorder width $\Delta$ at temperature $T / t=1 / 8$ with $U / t=1$ and $V / t=1$. 


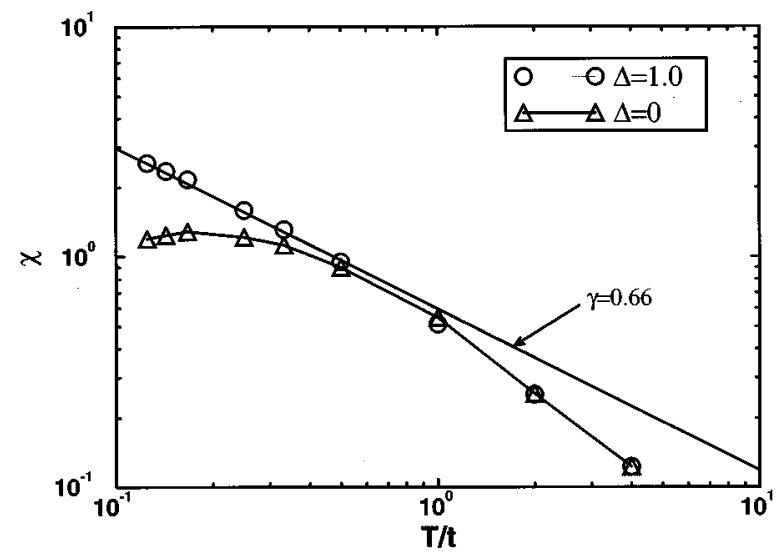

FIG. 2. Uniform $f$ magnetic susceptibility as a function of temperature for the hybridization-disorder width $\Delta=0$ (triangles) and $\Delta=1$ (circles) for $U / t=1$ and $V / t=1$. For $(T / t \leqslant 1), \chi$ can be scaled by the power law, $\chi$ $\sim T^{-\gamma}$, with $\gamma=0.66$, in excellent agreement with experiment (see Ref. 4).

leading to a singlet ground state. Turning on the disorder in hybridization results in a destruction of the singlet ground state and a reduction of the spin gap between the singlet ground state and the lowest lying excited triplet state. Figure 1 shows also that $C(i, i+1)$ increases monotonically with $\Delta$. These short-range $f-f$ antiferromagnetic correlations have been found to be as important as the $f$ - $c$ correlations in the formation of the singlet state in the PAM. ${ }^{7}$ Consistent with the above results, we find that increase of disorder in hybridization tends to suppress these short-range antiferromagnetic $f$ - $f$ correlations, resulting in noncompensated $f$ moments. On the other hand, $T \kappa$ is almost unchanged by disorder in hybridization. This suggests that the charge gap might be due to correlation effects rather than hybridization effects.

We present in Fig. 2 the $f$ magnetic susceptibility $\chi$ as a function of temperature for $\Delta=0$ (PAM) and $\Delta=1$. In contrast to the nondisordered case, $\chi$ exhibits a non-Fermi-liquid behavior, diverging at low temperatures. We find that $\chi$ can be scaled at low temperatures by a power law, $\chi \sim T^{-\gamma}$, with the exponent $\gamma=0.66$, which is in excellent agreement with recent experimental results. ${ }^{1}$ This result might be interpreted that the hybridization-disorder causes some of the local $f$ sites to possess low Kondo temperatures and consequently to behave as nearly free magnetic moments, leading to a NFL behavior.

We have also studied the effect of disorder in the $f$-electron energy level, $E_{f}$, on the magnetic properties, as

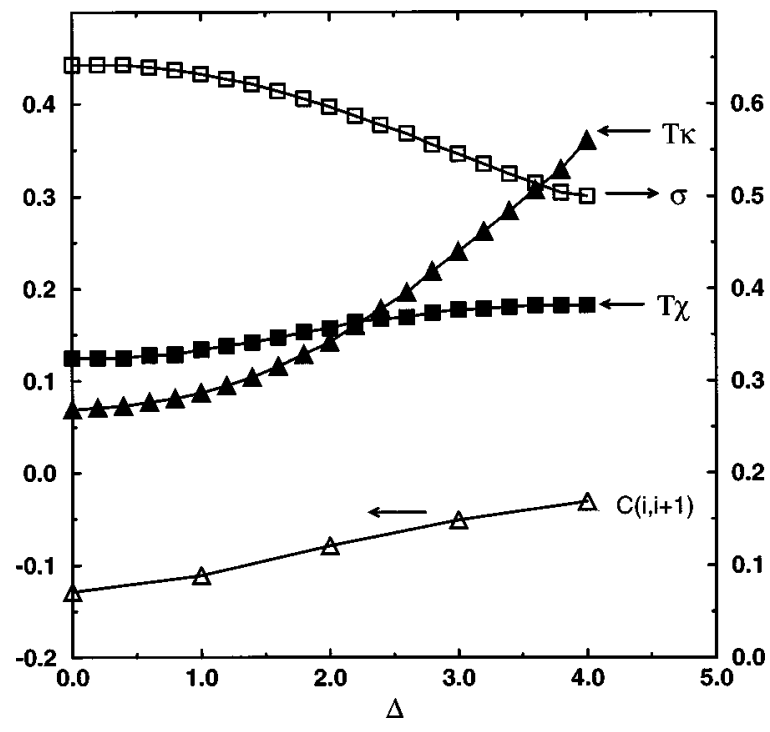

FIG. 3. Square of the $f$-orbital local moment $\sigma$ (open squares), the $f$ magnetic susceptibility $T \chi$ (solid squares), the charge susceptibility $T \kappa$ (solid triangles) and the nearest-neighbor $f-f$ spin correlation function $C(i, i+1)$ (open triangles) as a function of the energy-level-disorder width $\Delta$ at temperature $T / t=1 / 8$ with $U / t=1$ and $V / t=1$.

shown in Fig. 3. We find that in the weak-disorder regime, $\Delta / t \leqslant 1$, all quantities are almost unchanged by the disorder in $E_{f}$, in contrast to the case of disorder in hybridization. This is due to the fact that the majority of the $f$ sites are occupied, since the average $f$-electron energy level is below the Fermi energy $\left(-U_{f} / 2\right)$.

This work was supported through the National Science Foundation under Grant No. DMR-9531005 and the Office of Research and Sponsored Projects at California State University Northridge.

${ }^{1}$ M. C. de Andrade et al., cond-mat 9802081 (1998).

${ }^{2}$ B. Andraka and G. R. Stewart, Phys. Rev. B 47, 3208 (1993); B. Andraka, ibid. 49, 348 (1994).

${ }^{3}$ E. Miranda, V. Dobrosavljević, and G. Kotliar, Phys. Rev. Lett. 78, 290 (1997).

${ }^{4}$ A. Chattopadhyay and M. Jarrell, Phys. Rev. B 56, 2920 (1997).

${ }^{5}$ A. H. Castro Neto, G. Gastilla, and B. A. Jones, cond-mat/9710123.

${ }^{6}$ J. E. Hirsch and R. M. Fye, Phys. Rev. Lett. 56, 2521 (1986); J. E. Gubernatis, J. E. Hirsch, and D. J. Scalapino, Phys. Rev. B 35, 8478 (1987).

${ }^{7}$ R. Blankenbecler, J. R. Fulco, W. Gill, and D. J. Scalapino, Phys. Rev. Lett. 58, 411 (1987) 